Military Technical College

Kobry Elkobbah,

Cairo, Egypt

May 29-31,2012

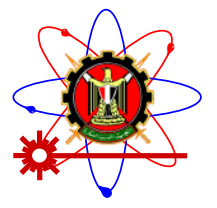

$6^{\text {th }}$ International Conference

on Mathematics and

Engineering Physics

(ICMEP-6)

\title{
Rough Set Theory Based on Two Relations and Its Applications
}

by

\author{
Arafa A. Nasef \\ Department of Physics and Engineering Mathematics, Faculty of Engineering, \\ Kafr El-Sheikh University, Kafr El-Sheikh, Egypt. E-mail: nasefa50@yahoo.com
}

\begin{abstract}
Rough sets theory was introduced by Pawlak in the early 80's and has reached a level of high visibility and maturity. In recent year's we have witness diverse as well as widespread research in rough sets theory and its applications worldwide.

In this paper, we introduce a new approximation space (biapproximation space) and define the lower and upper approximation based on two relations to take advantages of two relations in the same time. With this approximation and our definitions, we present two concepts to calculate the size of boundary region and discuss some of the basic properties of them. Examples are provided to illustrate the behavior of this new notion. Compared with Pawlak approximation space, our new approximation space is very efficient and settable when we have a lot of data for one case.
\end{abstract}

AMS Subject Classification: 54A10, 54E55.

Keywords and Phrases: Approximation space, rough set, bitopological spaces.

\section{Introduction}

The topological structure of a set is now considered as mathematical model for getting information from data $[1,2,7]$. The modeling process is based on relations obtained from a given data by one expert. Using two topologies help in discovering information using two points of view in the same time. Basic concepts of rough sets depends on a special type of topologies, namely quasi discrete topology, thus the general structures are generalizations of the quasi discrete topologies. Rough sets have been initiated by Pawlak $[8,9]$ in order to describe approximation knowledge of subsets of a given universe. In some sense this theory can be considered as a generalization of classical set theory and made a great success in knowledge acquisition in recent years and it has been applied in many applications such as knowledge discovery and machine learning $[5,6,11,14]$. Unfortunately, its based on 
Military Technical College

Kobry Elkobbah,

Cairo, Egypt

May 29-31,2012

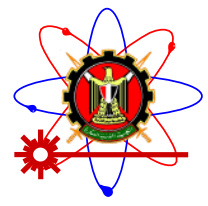

$6^{\text {th }}$ International Conference on Mathematics and Engineering Physics (ICMEP-6)

complete information systems, an approximation is pair $(U, R)$, where $U$ is a certain set called universe, and $R \subset U \times U$ is an equivalence relation based on ability to classify objects (elements of $U$ ) and it takes the form as real things, states, abstract concepts, moment of time, ..., etc and has equivalence classes and the set of all equivalence classes denoted by $U / R$, so the least (greatest) union of equivalence classes containing (contained in) set $X$ is the upper (lower) approximation which denoted by $H(X)(L(X))$. Now, any set in the approximation space is an exact or rough. By the use of two exact sets (lower and upper approximation), any rough set can be defined approximately in the approximation space.

\section{Biapproximation Space}

The aim of this section is to introduce the concept of a biapproximation space based on Pawlak approximation space and give examples to illustrate the behavior of this new notion.

Suppose $\mathrm{R}$ is a binary relation on a universe $\mathrm{U}$. Yao [14] defined a pair of approximation operations. $L(R), H(R): P(U) \rightarrow P(U)$ as follows:

$$
\begin{aligned}
& L(R)(X)=\{x: \forall y, x R y \Rightarrow y \in X\}=\{x: R N(x) \subseteq X\}, \\
& H(R)(X)=\{x: \exists y \in X, \text { s.t. } x R y\}=\{x: R N(x) \cap X \neq \phi\},
\end{aligned}
$$

where $R N(X)=\{y \in U: x R y\}$. They are called the lower approximation operation and the upper approximation operation, respectively.

Definition 2.1. Let $U$ be a finite set and $X \subset U, R_{1}, R_{2}$ be equivalence relations on $U$. Then

(i) $\pi=\left(U, R_{1}, R_{2}\right)$ is called a biapproximation space.

(ii) The bi-lower approximation of $X$ on $U$ is defined as $L_{\pi}(X)=L_{R_{1}}(X) U$ $L_{R_{2}}(X)$, where $L_{R_{i}}(X)=\bigcup\{Y \in U / R, Y \subset X\}, i \in\{1,2\}$.

(iii) The bi-upper approximation of $X$ on $U$ is defined as $H_{\pi}=H_{R_{1}}(X) \cap$ $H_{R_{2}}(X)$, where $H_{R_{i}}(X)=\bigcup\{Y \in U / R, Y \cap X \neq \phi\}, i \in\{1,2\}$.

Proposition 2.1. Let $\pi=\left(U, R_{1}, R_{2}\right)$ be a biapproximation space. Then the space $(U, R)$ which given by $U / R=\{X \cap Y: X \in U / R, Y \in U / R\}$, forms an expansion of both $\left(U, R_{1}\right)$ and $\left(U, R_{2}\right)$. 
Military Technical College

Kobry Elkobbah,

Cairo, Egypt

May 29-31,2012

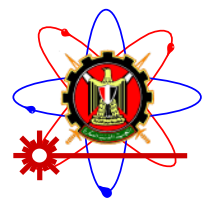

$6^{\text {th }}$ International Conference on Mathematics and Engineering Physics (ICMEP-6)

Proof. Since $(U, R)$ is finer than $\left(U, R_{1}\right)$ and $\left(U, R_{2}\right)$, then the proof is obvious.

Lemma 2.1. Let $\pi=\left(U, R_{1}, R_{2}\right)$ be a biapproximation space and $(U, R)$ be the expansion of $\left(U, R_{1}\right)$ and $\left(U, R_{2}\right)$. Then the following hold:

(i) $L_{\pi}(X) \subseteq L(X)$,

(ii) $H(X) \subseteq H_{\pi}(X)$,

where $L(X)$ and $H(X)$ are the lower and upper approximation of $X$ in the expansion $(U, R)$.

Proof. (i) Since $(U, R)$ is finer than $\left(U, R_{1}\right)$ and $\left(U, R_{2}\right)$. Then $L_{R_{1}}(X) \subseteq L(X)$ and $L_{R_{2}}(X) \subseteq L(X)$, then $L_{R_{1}}(X) \cup L_{R_{2}}(X) \subseteq L(X)$. Thus $L_{\pi}(X) \subseteq L(X)$.

(ii) Follows from Definition 2.1, and Proposition 2.1.

Remark 2.1. $L_{R_{i}}(X) \subseteq L_{\pi}(X) \subseteq L(X)$ and $H(X) \subseteq H_{\pi}(X) \subseteq H_{R_{i}}(X), i=1,2$. Proposition 2.2. Let $\pi=\left(U, R_{1}, R_{2}\right)$ be a biapproximation space and $(U, R)$ be the expansion. Then for $X \subset U$ the accuracy of $X$ with respect to the expansion which given by $\frac{|L(X)|}{|H(X)|}$ is greater than the accuracy of $X$ with respect to $\pi$ which given by $\frac{\left|L_{\pi}(X)\right|}{\left|H_{\pi}(X)\right|}$.

Proof. The proof is directly derivable from Lemma 2.1 and Remark 2.1.

Example 2.1. Let $\pi=\left(U, R_{1}, R_{2}\right)$ be a biapproximation space where $U=\{a, b, c$, $d, e, f, g, h\}, U / R_{1}=\{\{a, b, f\},\{c, d\},\{e, g, h\}\}, U / R_{2}=\{\{a, b, d\},\{c, e, h\}$, $\{f, g\}\}$ and $U / R=\{\{a, b\},\{c\},\{d\},\{e, h\},\{f\},\{g\}\}$. Since $X=\{a, b, d, g\}$, then $L(X)=\{a, b, d, g\}, L_{\pi}(X)=\{a, b, d\}, H(X)=\{a, b, d, g\}$ and $H_{\pi}(X)=$ $\{a, b, d, f, g\}$. Mean that $L_{\pi}(X) \subseteq L(X)$ and $H(X) \subseteq H_{\pi}(X)$.

The boundary region of any subset is contracted if it measured with respect to the biapproximation space (expansion of biapproximation space) and thus the degree of accuracy of two Pawlak approximation space increases. Also, we have the best results when we use the expansion of biapproximation space (Proposition 2.2).

\section{Relative Biapproximation Space}


Military Technical College

Kobry Elkobbah,

Cairo, Egypt

May 29-31,2012

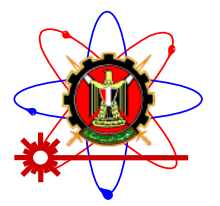

$6^{\text {th }}$ International Conference on Mathematics and Engineering Physics (ICMEP-6)

The aim of this section is to define the concept of lower and upper approximation of one classification of biapproximation space with respect to another classification and introduce new definitions indicate the useful of the relative biapproximation space.

Definition 3.1. Let $\pi=\left(U, R_{1}, R_{2}\right)$ be a biapproximation space and $U / R_{1}=\left\{X_{i}\right\}$ and $U / R_{2}=\left\{X_{j}\right\}$, where $i, j \in\{1,2, \ldots, n\}$. Then

(i) the lower approximation of $X$ on $U / R_{1}$ by using $U / R_{2}$ is

$$
L_{R_{1}}\left(X_{i}\right)=\bigcup\left\{X_{j} \in U / R_{2}, X_{j} \subset X_{i}\right\},
$$

(ii) the upper approximation of $X$ on $U / R_{1}$ by using $U / R_{2}$ is

$$
H_{R_{1}}\left(X_{i}\right)=\bigcup\left\{X_{j} \in U / R_{2}, X_{j} \cap X_{i} \neq \phi\right\} \text {. }
$$

Definition 3.2. Let $\pi=\left(U, R_{1}, R_{2}\right)$ be a biapproximation space where $U / R_{i}=$ $\left.X_{1}, X_{2}, \ldots, X_{n}\right\}, i \in\{1,2\}$ and $L_{R_{i}}\left(X_{j}\right)=\left\{L_{R_{i}}\left(X_{1}\right), L_{R_{i}}\left(X_{2}\right), \ldots, L_{R_{i}}\left(X_{j}\right)\right\}$ be the lower approximation of members of $U / R_{i}$ also the upper approximation of members of $U / R_{i}$ given by $H_{R_{i}}\left(X_{j}\right)=\left\{H_{R_{i}}\left(X_{1}\right), H_{R_{i}}\left(X_{2}\right), \ldots, H_{R_{i}}\left(X_{j}\right)\right\}$, $i, j \in\{1,2, \ldots, n\}$. Then,

(i) the accuracy of $U / R_{1}$ by $U / R_{2}$ defined as $\alpha_{R_{2}}\left(U, R_{1}\right)=\frac{\sum\left|L_{R_{i}}\left(X_{j}\right)\right|}{\sum\left|H_{R_{i}}\left(X_{j}\right)\right|}$,

(ii) the quality of $U / R_{1}$ by $U / R_{2}$ defined by $\gamma_{R_{2}}\left(U, R_{1}\right)=\frac{\sum\left|L_{R_{i}}\left(X_{j}\right)\right|}{|U|}$, where $j \in\{1,2, \ldots, n\}$.

Remark 3.1. The accuracy of $U / R_{1}$ expresses the percentage of possible correct decision when classifying objects employing the relation $R_{2}$ while the quality of $U / R_{1}$ expresses percentage of objects which can correctly classified to classes employing relation $R_{2}$.

Example 3.1. let $\pi=\left(U, R_{1}, R_{2}\right)$ be a biapproximation space, where $U=\{a, b, c$, $d, e, f, g, h\}, U / R_{1}=\{\{a, c, e\},\{b, d\},\{f, g, h\}\}$ and $U / R_{2}=\{\{a, b, d\},\{c, e, h\}$, $\{f, g\}\}$, then 
Military Technical College

Kobry Elkobbah,

Cairo, Egypt

May 29-31,2012 $6^{\text {th }}$ International Conference on Mathematics and Engineering Physics (ICMEP-6)

$$
\begin{aligned}
& \alpha_{R_{2}}\left(U, R_{1}\right)=\frac{0+0+2}{6+3+5}=\frac{2}{14}=\frac{1}{7} \approx 14 \%, \\
& \gamma_{R_{2}}\left(U, R_{1}\right)=\frac{0+0+2}{8}=\frac{2}{8}=\frac{1}{4}=25 \%, \\
& \alpha_{R_{1}}\left(U, R_{2}\right)=\frac{2+0+0}{5+6+3}=\frac{2}{14}=\frac{1}{7} \approx 14 \%,
\end{aligned}
$$

and

$$
\gamma_{R_{1}}\left(U, R_{2}\right)=\frac{2+0+0}{8}=\frac{2}{8}=\frac{1}{4}=25 \% .
$$

Example 3.2. Let $\pi=\left(U, R_{1}, R_{2}\right)$ be a biapproximation space, where $U=\{a, b, c$, $d, e, f\}, U / R_{1}=\{\{a\},\{b\},\{c, e\},\{d\},\{f\}\}$ and $U / R_{2}=\{a, b\},\{c\},\{d\}$, $\{e, f\}\}$, then

$$
\begin{aligned}
& \alpha_{R_{2}}\left(U, R_{1}\right)=\frac{0+0+1+1+0}{2+2+3+1+2}=\frac{2}{10}=\frac{1}{5}=20 \%, \\
& \gamma_{R_{2}}\left(U, R_{1}\right)=\frac{0+0+1+1+0}{6}=\frac{2}{6}=\frac{1}{3} \approx 33 \%, \\
& \alpha_{R_{1}}\left(U, R_{2}\right)=\frac{2+0+1+1}{2+2+1+3}=\frac{4}{7} \approx 57 \%,
\end{aligned}
$$

and

$$
\gamma_{R_{1}}\left(U, R_{2}\right)=\frac{2+0+1+1}{6}=\frac{4}{6}=\frac{2}{3} \approx 66 \% .
$$

Proposition 3.1. Let $\pi=\left(U, R_{1}, R_{2}\right)$ be a biapproximation space and $U / R_{i}=\left\{X_{1}\right.$, $\left.X_{2}, \ldots, X_{n}\right\}, i \in\{1,2\}$. Then the following hold:

(i) If there exists $j \in\{1,2, \ldots, n\}$ such that $L_{R_{1}}\left(X_{j}\right) \neq \phi$ then for each $k \neq j$ and $k \in\{1,2, \ldots, n\}, \quad H_{R_{1}}\left(X_{k}\right) \neq U \quad$ (the opposite is not true, if $L_{R_{i}}\left(X_{j}\right)=\phi$ is not true $\left.H_{R_{1}}\left(X_{k}\right)=U\right)$.

(ii) If there exists $j \in\{1,2, \ldots, n\}$ such that $H_{R_{1}}\left(X_{j}\right)=U$ then for each $k \neq j$ and $k \in\{1,2, \ldots, n\}, L_{R_{1}}\left(X_{k}\right)=\phi$ (the opposite is not true).

(iii) If for each $j \in\{1,2, \ldots, n\}, L_{R_{1}}\left(X_{j}\right) \neq \phi$ holds, then $H_{R_{1}}\left(X_{j}\right) \neq U$ for each $j \in\{1,2, \ldots, n\}$ (the opposite is not true). 
Military Technical College

Kobry Elkobbah,

Cairo, Egypt

May 29-31,2012

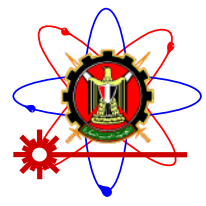

$6^{\text {th }}$ International Conference on Mathematics and Engineering Physics (ICMEP-6)

(iv) If for each $j \in\{1,2, \ldots, n\}, H_{R_{1}}\left(X_{j}\right) \neq \phi$ holds, then $L_{R_{1}}\left(X_{j}\right)=\phi$, for each $j \in\{1,2, \ldots, n\}$ (the opposite is not true).

Proof. (i) If $L_{R_{1}}\left(X_{j}\right) \neq \phi$, then there exists $X_{1} \in U / R_{2}\left(L_{R_{1}}\left(X_{j}\right)=\bigcup\left\{X_{1} \in U / R_{2}\right.\right.$, $\left.\left.X_{1} \subset X_{i}\right\}\right)$. For each $X_{k} \neq X_{j}, \quad H_{R_{1}}\left(X_{k}\right)=\bigcup\left\{X_{i} \in U / R_{2}, X_{i} \cap X_{k} \neq \phi\right\}$, $i \in\{1,2, \ldots, n\}$, then $H_{R_{1}}\left(X_{k}\right) \cap X_{1}=\phi$ and consequently $H_{R_{1}}\left(X_{k}\right) \neq U$ for each $j \neq k$. Or, if $L_{R_{1}}\left(X_{j}\right) \neq \phi$, then there exists $x \in X$ such that $x \in X \subset X_{j}$ which implies $X_{1} \cap X_{k}=\phi$ for each $j \neq k$. These yields $H_{R_{1}}\left(X_{R} \cap X_{i}\right)=\phi$ and $H_{R_{1}}\left(X_{k} \neq U\right)$, for each $j \neq k$.

(ii) If $U_{R_{1}}\left(X_{j}\right)=U\left(H_{R_{1}}\left(X_{j}\right)=\bigcup\left\{X_{i} \in U / R_{2}, X_{j} \cap X_{i} \neq \phi\right\}\right)$ that means $X_{j} \cap X_{i} \neq \phi$ for each $X_{1} \in U / R_{2}, i \in\{1,2, \ldots, n\}$, then $X_{i} \subset X_{k}$ doesn't hold or each $j \neq k,\left(\phi \subset X_{k}\right)$

$$
L_{R_{1}}\left(X_{k}\right)=\bigcup\left\{\phi \in U / R_{2}, \phi \subset X_{k}\right\} \text {, then } L_{R_{1}}\left(X_{k}\right)=\phi \text {. }
$$

(iii) and (iv) Follows similarly as in (i) and (ii).

Example 3.3. Let $\pi=\left(U, R_{1}, R_{2}\right)$ be a biapproximation space, where $U=\{a, b, c$, $d, e, f, g, h\}, U / R_{1}=\{\{a, c, e\},\{b, d\},\{f, g, h\}\}$ and $U / R_{2}=\{\{a, b, d\},\{c, e, h\}$, $\{f, g\}\}$, then $L_{R_{1}}(\{a, b, d\})=\{b, d\} \neq \phi$ and $H_{R_{1}}(\{c, e, h\})=\{a, b, d, f, g\} \neq U$ and $H_{R_{1}}(\{f, g\})=\{f, g, h\} \neq U$.

Example 3.4. Let $\pi=\left(U, R_{1}, R_{2}\right)$ be a biapproximation space, where $U=\{a, b, c$, $d, e, f, g, h\}$, with $U / R_{1}=\{\{a, c, e\},\{b, d\},\{f, g, h\}\}$ and $U / R_{2}=\{\{a, b, f\},\{c, d\}$, $\{e, g, h\}\}$, then $U_{R_{1}}(\{a, b, f\})=U, L_{R_{1}}(\{c, d\})=\phi$ and $L_{R_{1}}(\{e, g, h\})=\phi$.

\section{Bi-Equal and Bi-Inclusion}

The aim of this section is to define the concept of bi-equal and bi-inclusion of one classification of biapproximation space with respect to another classification.

Definition 4.1. Let $\pi=\left(U, R_{1}, R_{2}\right)$ be a biapproximation space and $X, Y \subset U$. Then the following hold:

(i) $X, Y$ are bottom equal on $U / R_{2}$ by $U / R_{1}$ if $L_{R_{1}}(X)=L_{R_{1}}(Y)$. 
Military Technical College

Kobry Elkobbah,

Cairo, Egypt

May 29-31,2012

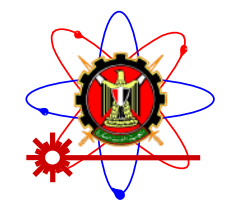

$6^{\text {th }}$ International Conference on Mathematics and Engineering Physics (ICMEP-6)

(ii) $X, Y$ are top equal $U / R_{2}$ by $U / R_{1}$ if $H_{R_{1}}(X)=H_{R_{1}}(Y)$.

(iii) $X, \quad Y$ are bi-equal on $U / R_{2}$ by $U / R_{1}$ if $L_{R_{1}}(X)=L_{R_{1}}(Y)$ and $H_{R_{1}}(X)=H_{R_{1}}(Y)$.

(iv) $X$ is bottom inclusion in $Y$ on $U / R_{2}$ by $U / R_{1}$ if $L_{R_{1}}(X) \subset L_{R_{1}}(Y)$.

(v) $X$ is top inclusion in $Y$ on $U / R_{2}$ by $U / R_{1}$ if $H_{R_{1}}(X) \subset H_{R_{1}}(Y)$.

(vi) $X$ is bi-inclusion in $Y$ on $U / R_{2}$ by $U / R_{1}$ if $L_{R_{1}}(X) \subset L_{R_{1}}(Y)$ and $H_{R_{1}}(X) \subset H_{R_{1}}(Y)$.

Definition 4.2. Let $\pi=\left(U, R_{1}, R_{2}\right)$ be a biapproximation space and $R_{1}$ and $R_{2}$ are equivalence relations. Then $R_{1}$ is a covering of $R_{2}$ if and only if $R_{2}$ depends on $R_{1}$ and $R_{1}$ is minimal (or, $R_{1}$ is covering of $R_{2}$ if and only if $R_{2}$ depends on $R_{1}$ and no proper subset $R_{1}^{*}$ of $R_{1}$ exists such that $R_{2}$ depends on $R_{1}^{*}$ ).

In other words, $R_{1}$ and $R_{2}$ are equivalence if $U / R_{1}=U / R_{2}$ (or, $R_{1}$ and $R_{2}$ have the same equivalence classes), and $R_{1}$ is finer than $R_{2}$ if $U / R_{1} \subset U / R_{2}$ or $R_{2}$ is coarser than $R_{1}$.

Example 4.1. Let $\pi=\left(U, R_{1}, R_{2}\right)$ be a biapproximation space, where $U=\{a, b, c$, $d, e, f\}$ with $U / R_{1}=\{\{a\},\{b\},\{c\},\{d\},\{e\},\{f\}\}$ and $U / R_{2}=\{\{a, b\},\{c, e, f\}$, $\{d\}$, then $R_{1}$ is covering of $R_{2}$ because $U / R_{1} \subset U / R_{2}$.

\section{Uniqueness of Binary Relations to Generate Rough Sets}

Theorem 5.1. Let $\pi=\left(U, R_{1}, R_{2}\right)$ be a biapproximation space and $X \subseteq U$. Then:

(i) $L\left(R_{1} \cup R_{2}\right)(X)=L\left(R_{1}\right)(X) \cap L\left(R_{2}\right)(X)$ and

(ii) $H\left(R_{1} \cup R_{2}\right)(X)=H\left(R_{1}\right)(X) \cup H\left(R_{2}\right)(X)$.

Proof. (i) For any $X \subseteq U, L\left(R_{1} \cup R_{2}\right)(X)=\left\{x: \forall y \in U, x\left(R_{1} \cup R_{2}\right) y\right.$ $\Rightarrow y \in X\}=\left\{x: \forall y \in U, x R_{1} y\right.$ or $\left.x R_{2} y \Rightarrow y \in X\right\}=\left\{x: \forall y \in U, x R_{1} y \Rightarrow y \in X\right\}$ $\bigcap\left\{x: \forall y \in U, x R_{2} y \Rightarrow y \in X\right\}=L\left(R_{1}\right)(X) \cap L\left(R_{2}\right)(X)$.

(ii) For any $X \subseteq U, H\left(R_{1} \cup R_{2}\right)(X)=\left\{x: \exists y \in X, x\left(R_{1} \cup R_{2}\right) y\right\}=\{x: \exists y \in X$, $x R_{1} y$ or $\left.x R_{2} y\right\}=\left\{x: \exists y \in X, x R_{1} y\right\} \bigcup\left\{x: \exists y \in X, x R_{2} y\right\}=H\left(R_{1}\right)(X) \cup H\left(R_{2}\right)(X)$. 
Military Technical College

Kobry Elkobbah,

Cairo, Egypt

May 29-31,2012

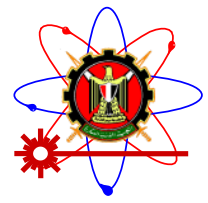

$6^{\text {th }}$ International Conference on Mathematics and Engineering Physics (ICMEP-6)

Proposition 5.1. Let $\pi=\left(U, R_{1}, R_{2}\right)$ be a biapproximation space. If $R_{1} \subseteq R_{2}$ then $L\left(R_{2}\right) \subseteq L\left(R_{1}\right)$ and $H\left(R_{1}\right) \subseteq H\left(R_{2}\right)$. Then we consider the situation for $R_{1} \cap R_{2}$.

Theorem 5.2. Let $\pi=\left(U, R_{1}, R_{2}\right)$ be a biapproximation space. Then:

(i) $L\left(R_{1}\right)(X) \cup L\left(R_{2}\right)(X) \subseteq L\left(R_{1} \cap R_{2}\right)(X)$ and

(ii) $H\left(R_{1} \cap R_{2}\right)(X) \subseteq H\left(R_{1}\right)(X) \cap H\left(R_{2}\right)(X)$.

Proof. It is easy to proof this theorem by Proposition 5.1.

The equalities in the above theorem is not hold generally as shown by the following example.

Example 5.1. Let $\pi=\left(U, R_{1}, R_{2}\right)$ be a biapproximation space, where $U=\{a, b, c\}$, $R_{1}=\{(a, a),(a, b),(b, b)\}, \quad$ and. $\quad R_{2}=\{(a, a),(a, c),(c, a),(c, b),(c, c)\}$. Then we have

$$
\begin{aligned}
& R N_{R_{1}}(\{a\})=\{a, b\}, R N_{R_{1}}(\{b\})=\{b\}, R N_{R_{1}}(\{c\})=\phi \\
& R N_{R_{2}}(\{a\})=\{a, c\}, R N_{R_{2}}(\{b\})=\phi, R N_{R_{2}}(\{c\})=\{a, b, c\} . \\
& R_{1} \cap R_{2}=\{(a, a)\}, \text { and } \\
& R N_{R_{1} \cap R_{2}}(\{a\})=\{a\}, R N_{R_{1} \cap R_{2}}(\{b\})=\phi, R N_{R_{1} \cap R_{2}}(\{c\})=\phi .
\end{aligned}
$$

For $X=\{a\}$ and $Y=\{b\}$, we have $L\left(R_{1}\right)(X)=\{c\}, H\left(R_{1}\right)(Y)=\{a, b\}$.

$$
\begin{aligned}
& L\left(R_{2}\right)(X)=\{b\}, H\left(R_{2}\right)(Y)=\{c\} \text {, and } \\
& L\left(R_{1} \cap R_{2}\right)(X)=\{a, b, c\}, H\left(R_{1} \cap R_{2}\right)(Y)=\phi .
\end{aligned}
$$

Thus, $L\left(R_{1}\right)(X) \cup L\left(R_{2}\right)(X) L\left(R_{1} \cap R_{2}\right)(X)$ and

$$
H\left(R_{1} \cap R_{2}\right)(Y) \subset H\left(R_{1}\right)(Y) \cap H\left(R_{2}\right)(Y) .
$$

Proposition 5.2. Let $\pi=\left(U, R_{1}, R_{2}\right)$ be a biapproximation space. If $H\left(R_{1}\right) \subseteq$ $H\left(R_{2}\right)$, then $R_{1} \subseteq R_{2}$.

Proof. For each $x, y \in U$, if $(x, y) \in R_{1}, y \in R N_{R_{1}}(x), x \in H\left(R_{1}\right)\{y\} \subseteq H\left(R_{2}\right)\{y\}$, so $R N_{R_{2}}(x) \cap\{y\} \neq \phi$, that means $(x, y) \in R_{2}$, thus $R_{1} \subseteq R_{2}$.

Corollary 5.1. Let $\pi=\left(U, R_{1}, R_{2}\right)$ be a biapproximation space. If $H\left(R_{1}\right)=H\left(R_{2}\right)$, then $R_{1}=R_{2}$. 
Military Technical College

Kobry Elkobbah,

Cairo, Egypt

May 29-31,2012

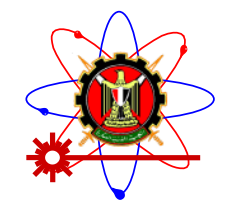

$6^{\text {th }}$ International Conference

on Mathematics and

Engineering Physics

(ICMEP-6)

Theorem 5.3. Let $\pi=\left(U, R_{1}, R_{2}\right)$ be a biapproximation space. Then $H\left(R_{1}\right)=$ $H\left(R_{2}\right)$ if and only if $R_{1}=R_{2}$.

Proof. It comes from Proposition 5.1 and Corollary 5.1.

By the duality between $H(R)$ and $L(R)$, we have the following result about $L(R)$.

Proposition 5.3. Let $\pi=\left(U, R_{1}, R_{2}\right)$ be a biapproximation space. If $L\left(R_{1}\right) \subseteq$ $L\left(R_{2}\right)$, then $R_{2} \subseteq R_{1}$.

Corollary 5.2. Let $\pi=\left(U, R_{1}, R_{2}\right)$ be a biapproximation space. If $L\left(R_{1}\right)=L\left(R_{2}\right)$, then $R_{1}=R_{2}$.

Theorem 5.4. Let $\pi=\left(U, R_{1}, R_{2}\right)$ be a biapproximation space. Then $L\left(R_{1}\right)=$ $L\left(R_{2}\right)$ if and only if $R_{1}=R_{2}$.

\section{Conclusions}

Pawlak approximation space is considered as mathematical model for getting information from data. The modeling process is based on equivalence relation obtained from a given data by one expand (view). Using two Pawlak approximation spaces (Biapproximation space) help in discovering information using two points of views in the same time and the new of views in the same and the new approximation focus on the expansion of the original model proposed by Pawlak. So the purpose of this paper is to extend the concept of lower and upper approximation. Pawlak approximation space introduced the diagnosis (solution) of some problems in Math., Chemistry, ..., etc but when we have two (,any) diagnosis's for any problem we use biapproximation space to find the best diagnosis or study one of these diagnosis's by using another one. Compared with Pawlak approximation space, our new approximation space is very efficient and settable when we have a lot of data for one case.

We proved that two different binary relations will generate two different lower approximation operations and two different upper approximation operations. As far as the applications of binary relation based rough sets to knowledge discovery from database are concerned, the reader is referred to $[3,4,10]$. In this future, we will explore the relationships between binary relation based rough sets and covering based 
Military Technical College

Kobry Elkobbah,

Cairo, Egypt

May $29-31,2012$

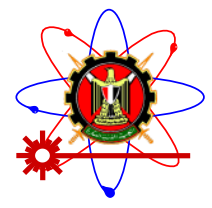

$6^{\text {th }}$ International Conference on Mathematics and Engineering Physics (ICMEP-6)

rough sets [15]. Another future research topic is to apply binary relation based rough set theory to the computational theory of linguistic dynamic systems [12] and security $[16,17]$.

\section{References}

[1] B. P. Dvalishvili, The lower and Upper Topologies as Bitopological. Proc. Tbilisi State Univ. 55, (2003), 37-52.

[2] R. Koppermqn and J. D. Lawson, Bitopological and Topological ordered $k$-spaces. Topology and its Applications 146-147, (2005), 385-396.

[3] M. Kryszkiewicz, Rough set approach to incomplete information systems, Information Sciences 112 (1998), 39-49.

[4] M. Kryszkiewicz, Rule in incomplete information systems, Information Sciences 113 (1998), 271-292.

[5] T. Y. Lin and N. Cercone, Rough Sets and Data Mining Analysis of Imprecies Data, Kluwer Academic Publisher, 1997.

[6] T. Y. Lin and Y. Y. Yao, Data Mining, Rough sets and Granular Computing, Physica-Verlag, 2002.

[7] T. Y. Lin, Neighbourhood Systems Information Granulation in P. P. Wong (Ed) Joint Conference of Information Sciences, March 1-5, Duch University, Vol. 3, 161-164, 2001.

[8] Z. Pawlak, Rough Sets. Ics Reports (431) (1981).

[9] Z. Pawlak, Rough Sets. International Journal of Information and Computer Science, 11, (1982), 341-356.

[10] R. Slowinski and D. Vanderpooten, A generalized definition of rough approximations based on similarity, IEEE Transactions on Knowledge and Data Engineering 12 (2) (2000), 331-336.

[11] R. Swiniarski, Rough Sets Methods in Feature Reduction and Classification. Int. H. Appl. Math. Computer Sci., Vol. 3, (2001), 565-582.

[12] F. Y. Wang, On the abstraction of conventional dynamic systems: from numerical analysis to linguistic analysis, Information Sciences 171 (1-3) (2005), 233-259. 
Military Technical College

Kobry Elkobbah,

Cairo, Egypt

May 29-31,2012

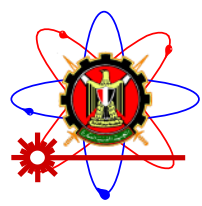

$6^{\text {th }}$ International Conference on Mathematics and Engineering Physics (ICMEP-6)

[13] Hu. Xiaohua; Han Jianchau and T. Y. Lin, New Rough Sets Model Based on Database System, Fundamneta Informatica (2004).

[14] Y. Yao, Constructive and algebraic methods of theory of rough sets, Information Sciences 109 (1998), 21-47.

[15] W. Zhu and F. Y. Wang, Reduction and axiomization of covering generalized rough sets, Information Sciences 152 (2003), 217-230.

[16] W. Zhu and F. Y. Wang, Covering based granular computing of conflict analysis, in IEEE ISI 2006, LNCS, Vol. 3975, (2006), 566-571.

[17] W. Zhu, Topological approaches to covering rough sets, Information Sciences 177 (6) (2007), 1499-1508. 